Noname manuscript No.

(will be inserted by the editor)

\title{
Understanding the role of open peer review and dynamic academic articles
}

Authors' reply to "Problems with natural selection of academic papers"

Pandelis Perakakis · Michael Taylor • Marco

G. Mazza - Varvara Trachana

Received: date / Accepted: date L. Egghe that we believe are inaccurate or require further comment.
P. Perakakis
Department of Personality, Evaluation and Psychological Treatment
University of Granada
Campus Cartuja, 18071
Granada
Spain
Tel.: +34-958 243753
E-mail: peraka@ugr.es

Abstract We welcome the commentary by L. Egghe [1] stimulating discussion on our recent article "Natural selection of academic papers" (NSAP) [2] that focuses on an important modern issue at the heart of the scientific enterprise - the open and continuous evaluation and evolution of research. We are also grateful to the editor of Scientometrics for giving us the opportunity to respond to some of the arguments by 
Keywords academic publishing $\cdot$ open peer review

L. Egghe claims that our article presents an unbalanced criticism of the current peer review (PR) system and that it negatively associates PR with commercial publishing. The aim of our article was not to provide a detailed description of academic publishing and the PR process. For details on these subjects we direct the interested reader to a review article published in UNESCO's 2010 World Social Science Report [3], where we present a brief, but comprehensive account of current academic publishing practices accompanied by recent statistics. Instead, in NSAP we explained how the key problematic elements of the present publishing system and the PR process can be adequately addressed by an alternative model based on open and transparent PR. In his commentary however, L. Egghe describes a distorted and somewhat inaccurate version of the academic publishing landscape.

Firstly, L. Egghe claims that scientific communication is already performed via two channels: (1) pre-print publication to online open-access repositories, and (2) submission to peer reviewed academic journals. Unfortunately however, evidence suggests that the practice of self-archiving is not nearly as widespread as L. Egghe suggests. According to a recent investigation [4], only $39 \%$ of authors surveyed had self-archived at least one of their articles. Furthermore, while over $90 \%$ of academic journals now permit selfarchiving, it is estimated that no more than 10-20\% of published articles have actually been self-archived [5]. This is exacerbated in the fields of social sciences and humanities where authors are less familiar with self-archiving practices. As a result, repositories in these areas trail those of other academic disciplines in rates of establishment and article submission [6]. Furthermore, the picture painted by searches for academic articles online with scientometric tools like Google Scholar is also bleak, since in the vast majority of cases articles are not self-archived and the reader is directed to the journal's 
publisher website where full-text versions are only available at a high price. Therefore, even though self-archiving exists, actual figures do not support the optimistic claim that scientific communication is being performed via pre-print publication to online open-access repositories in parallel with submission to peer reviewed academic journals. For this reason, in NSAP we clearly state that mandated self-archiving is required as part of the solution to the problem of accessibility to academic knowledge.

Secondly, L. Egghe argues that the current publishing model poses no significant accessibility barrier to developing countries. However, published statistics $[7,8]$ and articles expressing the outrage by academics from developing countries [9], suggest otherwise. Charitable initiatives like HINARI and AGORA resemble attempts to treat infectious diseases with painkillers that only temporarily distract attention from the root cause of suffering. As long as such initiatives depend on publisher agreements, donations and subsidies, their long-term sustainability is highly-questionable [10]. According to reports by academics from developing countries, what is needed is a real cure that inevitably involves disentangling the evaluation of scientific articles from commercial interests [11].

Thirdly, L. Egghe challenges our assertion that PR is controlled by the publishing industry. He correctly identifies that journal editors — rather than publishers — are responsible for assigning articles to reviewers. However, it is not true that PR is situated outside the commercial publishing industry. Instead, the PR process that determines the evaluation of academic articles and consequently academics themselves is the last bastion of control over science by commercial publishers. Other paid-for services such as, production of copy, distribution and intellectual copyright can now be efficiently handled by authors themselves. For this reason, the PR process operated by journals remains heavily biased by commercial and socio-political interests of the publishers [12]. 
An example of that is provided by L. Egghe's notion that a reviewer's role is to predict whether or not the article under examination will be "qualified" in the future, i.e. that it will receive many citations. This exposes the true concern of many journal editors, which is to publish research that will be highly-cited and therefore increase the impact factor of their journals. Science however, has not entrusted journal editors or reviewers with the task of judging quality based on projections of an article's future citation impact. The true judgement of academic quality, which should be a reviewer's only task, involves the assessment of a whole host of parameters not necessarily reflected in citation ratings [13]. In general, the current academic evaluation system based on citation metrics and a blind PR process, permits unethical conduct from all involved parties; editors, authors and reviewers $[14,15,12]$. We strongly believe that the natural selection model provides a satisfying alternative by ensuring free-for-all access to all academic articles, and more importantly, by providing a sustainable evaluation system exempt from commercial or other individual interests.

Finally, in his commentary, L. Egghe raises some important questions regarding the functioning of an open PR system, that we wish to address here.

\section{Open PR}

Open PR is not a new idea and many scholars already support that it is a viable solution to the numerous deficiencies of the current evaluation system $[16,17]$. Our model proposes a fully-transparent PR process, whereby reviews by referees are posted online and tagged to the article in question. This allows the implementation of a reviewer evaluation system that will provide motivation for potential reviewers [18]. Referee efforts will be acknowledged and rewarded, thus enhancing their academic standing 
in the field. Such a mechanism will also help to solve the "difficult to find reviewers" and "time limitation of the reviewers" problems mentioned by L. Egghe. In our system, writing a good review could be scientifically as beneficial as contributing an original article. Furthermore, open PR will sidestep other serious concerns too, such as reviewers evaluating papers outside their area of expertise, or writing a positive review as a favour, since their review will be subject to open criticism from the scientific community.

While L. Egghe holds that PR is guided by deontological rules, this is something that is not transparent in a blind PR system. On the contrary, open PR creates a public environment where everyone can judge and where every judgment can be weighted. The NSAP model turns what constitutes a weakness for classical journals into a strength for science and society. In the open environment we advocate, a large scientific community can assess the quality of a manuscript, or even discover frauds or sources of plagiarism a lot more efficiently than is possible today by two or three anonymous and unmotivated reviewers. The efficiency of social networks improves as the number of participating actors, and more importantly, their interconnectedness grows. It is expected, therefore, that as more and more scholars submit reviews that start to build an interconnected network, a natural consensus on the quality and relevance of submitted manuscripts is bound to emerge. As the creator of open source Linux, Linus Torvalds said, "given enough eyeballs, all bugs are shallow" [19].

In the current academic publishing system only about one in ten articles ever receive a single citation. As a result, the vast majority of scientific communications remain in the dark since being cited by other articles is a main source of visibility. However, authors are not always guided by quality when deciding which articles to cite, but are rather influenced by other irrelevant criteria, such as an article's impact factor. The 
open PR system presents the author with the opportunity to address the problem of visibility by being the driving force seeking reviewers and reviews, which will likely also increase article citation rates. Here, we disagree with L. Egghe that bias will be introduced if authors drive the PR process, since in a transparent system the academic community as a whole will be able to regulate both positive and negative evaluation trends driven by personal rather than objective scientific criteria. That this is likely to be the case is reflected by other online communities that perform collective editing such as Wikipedia or the blogosphere, where it is well known that the "Wisdom of crowds" is an efficient mechanism in controlling bias [20].

\section{Article Timelines and Article Threads}

In the NSAP model, an article, like the academic ideas upon which is it based, is an amorphous entity that mutates, breeds and evolves. This raises a reasonable question: at what moment should an article receive judgement or acceptance? In the view of science as a dynamical process, there are no clear and definite judgements. New findings overturn old, and past theories continuously get supplanted by new ones. Evaluation timelines are artificially imposed by journals to serve the need to assess individual articles and scientists. In this static evaluation system, PR is the last checkpoint of an article's academic quality. Flaws and weaknesses that pass through PR are rarely recognized and corrected post-publication. In the meantime, their authors can enjoy the benefits of having an impact factor publication, which another system would have righteously rejected. The recent "trial by twitter" [21], reflects the scientific community's need for a mechanism that enables open evaluation of science. The NSAP model allows constant assessment of academic articles, while at the same time any committee 
can evaluate individual articles or scientists based on their performance at the moment of the evaluation.

Furthermore, our NSAP model promotes a view of the article like a computer application software that evolves by continuously improving on and updating previous versions. In the case of open source computer software all versions of the program are located in the same webpage and users are always certain that they are using the latest and most efficient version. All information is gathered at the same place, the history of the update record is registered and the copyright of the creator protected. The application of a similar system to academic articles would lead to a gradual reduction in the "sea of un-cited articles" and the inadvertent repetition of ideas. Moreover, all article reviews and other relevant comments would be concentrated in the same location and readily available to all readers. For example, in the current debate on the NSAP model, all readers of our original article would also access L. Egghe's commentary, as well as this reply. Unfortunately, this is something that is unlikely to occur in the current publishing system.

\section{Immediate Implementation}

An important thing to note is that the natural selection model can be implemented right away in parallel to classical journal submission. It does not require any structural changes, just linked online repositories that accept and perform open PR. All that is needed is a quantitative meta-data tool to implement and update reviewer ratings. The Open Archives Initiative Protocol for Meta-data Harvesting (OAI-PMH) is an example of an existing tool that could be modified to include quantitative measures of referee performance. 


\section{Conclusion}

Once upon a time, authors entrusted journals with the job of guaranteeing academic quality through peer-review as well producing and distributing high quality copy. Today, they submit to ISI journals out of obligation, in the hope of raising the impact factor of their CVs. The question authors should ask is this: what is the added value that journals give to academic articles that justifies prohibitive publication fees, extortionate journal bundle subscription costs, and four figure open access options? With LaTeX templates, online repositories, an open PR system, and scientometrists and librarians on their side, authors can now do the job themselves for free, faster, and with a higher chance of being cited. Commercial journals are no longer the fittest species on the academic landscape, and as Darwin taught us, only the fittest survive. The question is not if, but when, natural selection of academic papers will become a reality.

\section{References}

1. Egghe, L. Problems with natural selection of academic papers. Scientometrics. current issue [to be completed by the editor].

2. Perakakis P, Taylor M, Mazza M, Trachana V. Natural selection of academic papers. Scientometrics. 2010;85(2):553-559.

3. Perakakis P, Taylor M, Mazza M, Trachana V. The roads to open access. In: World Social Science Report 2010. UNESCO; 2010. p. 307-309.

4. Swan A, Brown S. Authors and open access publishing. Learned publishing. 2004;17(3):219-224.

5. Harnad S, Brody T, Vallières F, Carr L, Hitchcock S, Gingras Y, et al. The access/impact problem and the green and gold roads to open access: An update. Serials review. 2008;34(1):36-40.

6. Xia J. Disciplinary repositories in the social sciences. In: ASLIB Proceedings New Information Perspectives. vol. 59. London: Aslib.; 2007. p. 528-538. 
7. Chan L, Costa S. Participation in the global knowledge commons: challenges and opportunities for research dissemination in developing countries. New Library World. $2005 ; 106(3 / 4): 141-163$.

8. Evans JA, Reimer J. Open access and global participation in science. Science. $2009 ; 323(5917): 1025$.

9. Barcinski MA. Disruption to science in developing countries. Nature. 2003;423(6939):480480.

10. Kirsop B, Chan L. Transforming access to research literature for developing countries. Serials Review. 2005;31(4):246-255.

11. Habib A. Challenging the international academic publishing industry. In: World Social Science Report 2010. UNESCO; 2010. p. 311.

12. Taylor M, Perakakis P, Trachana V. The siege of science. Ethics in Science and Environmental Politics(ESEP). 2008;8(1):17-40.

13. Moed HF. Citation analysis in research evaluation. Kluwer Academic Pub; 2005.

14. Seglen PO. Why the impact factor of journals should not be used for evaluating research. Bmj. 1997;314(7079):497.

15. Scully C, Lodge H. Impact factors and their significance; overrated or misused? British dental journal. 2005;198(7):391-393.

16. Gura T. Scientific publishing: Peer review, unmasked. Nature. 2002;416(6878):258-260.

17. Godlee F. Making reviewers visible. JAMA: the journal of the American Medical Association. $2002 ; 287(21): 2762$.

18. Wilson R. 'Referee factor' would reward a vital contribution. Nature. 2006;441(7095):812812.

19. Raymond ES. The Cathedral \& the Bazaar. O'Reilly; 1999.

20. Lehmann S, Jackson A, Lautrup B. Life, death and preferential attachment. EPL (Europhysics Letters). 2005;69:298.

21. Mandavilli A. Trial by twitter. Nature. 2011;469:286-287. 